\title{
EDITORIAL \\ Deep brain stimulation for tinnitus: exploring the frontier between sensory perception and awareness
}

\author{
Martin Jakobs, MD, ${ }^{1,2}$ and Andres M. Lozano, MD, $\mathrm{PhD}^{1}$ \\ 1Division of Neurosurgery, Toronto Western Hospital, University Health Network, Toronto, Ontario, Canada; and 2Department of \\ Neurosurgery, University Hospital Heidelberg, Germany
}

$\mathrm{T}$ INNITUS is the perception of sound when no actual external sound is present, and is therefore regarded as a phantom sensation comparable with phantom limb pain. It is a common disorder in industrialized countries, especially when levels of exposure to noise in professional or private life are high.

Peripheral hearing loss with reduced cochlear function is often present when tinnitus develops. Reduced input from the cochlear system is thought to cause central auditory system deafferentation, hyperactivity, and hyperexcitability. ${ }^{2,4}$ Lack of input in thalamic neurons causes hyperpolarization, burst firing, and pathological gamma band oscillations, and overall thalamocortical dysrhythmia., 5 It has been observed that the primary auditory cortex also shows maladaptive neuroplasticity with reorganized frequency maps (tonotopy), focal hyperactivity, neuronal synchrony, burst firing, and gamma-theta phase-amplitude coupling. ${ }^{5,6,13}$ These features resemble alterations found in other circuitopathies like movement disorders or chronic pain - both of which are amenable to surgical neuromodulation.

One structure that may act as a bridging point between the auditory and the limbic system and therefore between the sensorineural perception of sounds and the awareness and emotional charge of said percepts is the dorsal striatum including the locus of caudate neurons (area LC).

The current article "Phase I trial of caudate deep brain stimulation for treatment-resistant tinnitus" by Cheung et al. ${ }^{3}$ represents the first phase I trial of continuous bilateral deep brain stimulation (DBS) in area LC for treatmentresistant tinnitus.

Area LC represents the transition zone between the head and body of the caudate and it came to the group's attention after a patient with Parkinson disease (PD) and coexisting chronic bilateral tinnitus underwent left-sided subthalamic nucleus DBS lead placement and reported markedly reduced phantom percepts in both ears the day after surgery; this occurred without any stimulation. ${ }^{7}$ Post- operative imaging showed a stroke-like vascular injury medial to the DBS lead in the head and anterior portion of the body of the caudate. The patient remained tinnitus free in the ipsilateral ear and with substantially reduced phantom percepts in the contralateral ear for the follow-up duration of 18 months.

This and a published case of elimination of a preexisting bilateral tinnitus due to a left-sided ischemic lacunar stroke in the body of the caudate and the bordering white matter tracts without changes in overall hearing ${ }^{10}$ spawned interest in assessing the possibility of neuromodulation of the caudate to treat tinnitus. Targeting the caudate with DBS was thought to be easily achievable because typical trajectories of DBS leads for common movement disorder targets such as the subthalamic nucleus run close to the caudate or even traverse it.

The current article investigates whether bilateral continuous caudate DBS has meaningful beneficial effects in a population of patients that suffers severely from tinnitus and objectively investigates hearing safety before and after the procedure. ${ }^{3}$ Five of 6 patients were able to successfully complete the study protocol with a $60 \%-80 \%$ response rate regarding the Tinnitus Functional Index and Tinnitus Handicap Inventory after 24 weeks of DBS on the most effective stimulation settings.

Consistent with previous findings, continuous caudate DBS did not significantly alter hearing thresholds, thereby preserving hearing safety. No surgical complications or permanent stimulation-related side effects were observed.

To further refine targeting, the authors applied microelectrode recording and macrostimulation in up to 3 tracts spanning an area of up to $10 \mathrm{~mm}$ along the anterior-posterior axis of the caudate to define the optimal position for the implant. In a recent publication that includes the 6 patients from this phase I study, a more posterior position of implanted leads was associated more often with acute tinnitus loudness reduction during acute stimulation. ${ }^{11}$ This area located more in the body of the caudate comprises 
the section of -8 to $-15 \mathrm{~mm}$ in MNI (Montreal Neurological Institute) space. These caudate body subdivisions also showed a stronger functional connectivity to the auditory cortex compared to the caudate head.

Given the size of the nucleus, further refinement of the most effective target within the caudate is warranted and a preoperative functional connectivity analysis may be a feasible option to do so.

The study protocol allowed the group to find the optimal stimulation parameters before entering the 24-week stimulation period. This led to two distinct findings: 1) stimulation parameters (including frequency, pulse width, and amplitude) varied greatly between patients; and 2) the process of finding the optimal stimulation parameters was a lengthy one (5-13 months). ${ }^{3}$ The authors speculate that phantoms of different qualities may respond to different stimulation frequencies. This can only be proven within a bigger study population in which the tonal qualities of the phantoms are defined and compared to effective stimulation parameters or to the area of strongest functional connectivity within the caudate.

It seems tempting to record local field potentials from the DBS electrodes to find a neurophysiological biomarker (such as a decrease in the power spectrum of gamma oscillations) that coincides with phantom loudness reduction and to then apply closed-loop DBS to adjust stimulation settings accordingly, as has been investigated in PD patients. ${ }^{8,9}$ Furthermore, studies of transcranial and cortical stimulation found differential effects on tinnitus loudness when applying either tonic, burst, or noise stimulation. ${ }^{4,15}$ However, current commercially available DBS systems only allow for open-loop tonic stimulation and therefore have technical limitations that need to be overcome in the future.

The question whether a certain level of emotional distress or presence of psychiatric comorbidities would favor neuromodulation of the ventral striatum/limbic system rather than the dorsal striatum cannot currently be answered.

Overall, a recent study has evaluated the willingness of patients suffering from tinnitus to undergo invasive treatments. Approximately $20 \%$ of patients would fully accept DBS as an invasive treatment if it was able to cure tinnitus with an at least $50 \%$ chance, whereas $60 \%$ would not accept DBS. ${ }^{14}$ These rates were lower than for hearing aids (control group) but did not differ significantly from established treatments such as cochlear implants. Patients with previously failed treatment attempts showed an overall higher acceptance rate for invasive treatments. These findings demonstrate a certain place and demand for invasive neuromodulatory treatments for chronic tinnitus in a subset of patients.

It remains unclear how high acceptance rates for incision- and implant-free lesional neuromodulation techniques such as focused ultrasound (FUS) might be. Given that the interest in the caudate for treatment of tinnitus was sparked by unilateral stroke lesions and their sustained bilateral effect on auditory phantoms, FUS may represent a potential future treatment option to be explored, because functional and structural neuroimaging has been shown to help refine targeting.
The group around Cheung and Larson have successfully worked on the concept of caudate DBS for treatmentresistant tinnitus. Starting with the incidental finding of a caudate lesion after DBS implantation for movement disorders suppressing tinnitus, the group was able to translate this concept with pilot trials in patients with movement disorders to the first ever prospective phase I trial of DBS in patients with tinnitus. Although questions on how to define the optimal target and stimulation settings remain and may only be answered in a bigger phase II trial, this effort represents an important contribution in the field of surgical neuromodulation.

https://thejns.org/doi/abs/10.3171/2019.4.JNS191023

\section{References}

1. Boutet A, Ranjan M, Zhong J, Germann J, Xu D, Schwartz ML, et al: Focused ultrasound thalamotomy location determines clinical benefits in patients with essential tremor. Brain 141:3405-3414, 2018

2. Cheung SW, Larson PS: Tinnitus modulation by deep brain stimulation in locus of caudate neurons (area LC). Neuroscience 169:1768-1778, 2010

3. Cheung SW, Racine CA, Henderson-Sabes J, Demopoulos C, Molinaro AM, Heath S, et al: Phase I trial of caudate deep brain stimulation for treatment-resistant tinnitus. J Neurosurg [epub ahead of print September 24, 2019. DOI: 10.3171/2019.4.JNS19347]

4. De Ridder D, Vanneste S, van der Loo E, Plazier M, Menovsky T, van de Heyning P: Burst stimulation of the auditory cortex: a new form of neurostimulation for noise-like tinnitus suppression. J Neurosurg 112:1289-1294, 2009

5. Donovan C, Sweet J, Eccher M, Megerian C, Semaan M, Murray G, et al: Deep brain stimulation of Heschl gyrus: implantation technique, intraoperative localization, and effects of stimulation. Neurosurgery 77:940-947, 2015

6. Larson PS, Cheung SW: Deep brain stimulation in area LC controllably triggers auditory phantom percepts. Neurosurgery 70:398-406, 2012

7. Larson PS, Cheung SW: A stroke of silence: tinnitus suppression following placement of a deep brain stimulation electrode with infarction in area LC. J Neurosurg 118:192-194, 2013

8. Little S, Beudel M, Zrinzo L, Foltynie T, Limousin P, Hariz $\mathrm{M}$, et al: Bilateral adaptive deep brain stimulation is effective in Parkinson's disease. J Neurol Neurosurg Psychiatry 87:717-721, 2016

9. Little S, Brown P: The functional role of beta oscillations in Parkinson's disease. Parkinsonism Relat Disord 20 (Suppl 1):S44-S48, 2014

10. Lowry LD, Eisenman LM, Saunders JC: An absence of tinnitus. Otol Neurotol 25:474-478, 2004

11. Perez PL, Wang SS, Heath S, Henderson-Sabes J, Mizuiri D, Hinkley LB, et al: Human caudate nucleus subdivisions in tinnitus modulation. $\mathbf{J}$ Neurosurg [epub ahead of print February 8, 2019. DOI: 10.3171/2018.10.JNS181659]

12. Rammo R, Ali R, Pabaney A, Seidman M, Schwalb J: Surgical neuromodulation of tinnitus: a review of current therapies and future applications. Neuromodulation [epub ahead of print], 2018

13. Shi Y, Burchiel KJ, Anderson VC, Martin WH: Deep brain stimulation effects in patients with tinnitus. Otolaryngol Head Neck Surg 141:285-287, 2009

14. Smit JV, Pielkenrood BJ, Arts RAGJ, Janssen ML, Temel Y, Stokroos RJ: Patient acceptance of invasive treatments for tinnitus. Am J Audiol 27:184-196, 2018

15. Vanneste S, Fregni F, De Ridder D: Head-to-head compari- 
son of transcranial random noise stimulation, transcranial AC stimulation, and transcranial DC stimulation for tinnitus. Front Psychiatry 4:31-33, 2013

\section{Disclosures}

Dr. Lozano is a consultant for Medtronic, St. Jude, and Boston Scientific.

\section{Correspondence}

Andres M. Lozano: andres.lozano@uhnresearch.ca.

INCLUDE WHEN CITING

Published online September 24, 2019; DOI: 10.3171/2019.4.JNS191023.

\section{Response}

\section{Steven W. Cheung, MD, ${ }^{1,2}$ and Paul S. Larson, MD2,3}

1Department of Otolaryngology-Head and Neck Surgery, University of California, San Francisco; ${ }^{2}$ Surgical Services, Veterans Affairs Health Care System, San Francisco; and ${ }^{3}$ Department of Neurological Surgery, University of California, San Francisco, California

Drs. Jakobs and Lozano have kindly written a critical précis of our first-in-human phase I clinical trial of bilateral caudate nucleus neuromodulation by DBS for severe, treatment-resistant tinnitus. Their thoughtful editorial highlights considerations that should be entertained in future clinical trials.

Targeting refinement of the caudate nucleus by using the strength of auditory corticostriatal functional connectivity is a promising strategy that may ultimately standardize a treatment-responsive, biomarker-based approach to shorten time to benefit and increase treatment effect size for both implant-dependent and implant-free neuromodulation approaches. In cohort contrast studies, increased corticostriatal connectivity between the caudate nucleus body subdivision and auditory cortex has been demonstrated for tinnitus with bilateral hearing loss ${ }^{4}$ and bothersome tinnitus of at least moderate severity. ${ }^{9}$ The translation of cohort differences in resting-state fMRI auditory corticostriatal functional connectivity maps to individual-specific, personalized functional connectivity maps that would serve as a clinical tool for more precise caudate targeting will require innovative development and rigorous validation.

Although caudate targeting based on auditory corticostriatal functional connectivity may ultimately prove to be a useful method, dorsal striatal subsite neuromodulation may fail to deliver suitable benefit for those distressed patients with tinnitus whose disorder's severity is primarily driven by neuropsychiatric comorbidities or limbic cortex, or an exceptionally dysfunctional auditory cortex. Here, the addition of ventral striatal/limbic cortex neuromodulation by DBS or auditory cortical neuromodulation performed using intracranial or transcranial techniques may yield further benefit to achieve clinical success. Our phantom percepts striatal gate-control model ${ }^{5}$ accounts for those and other tinnitus modulators, and provides a conceptual anchor to target them to enhance treatment benefit.

This trial recruited highly distressed patients with tinnitus who had exhausted conventional therapies and were willing to accept invasive surgical neuromodulation without a priori knowledge of treatment effect size. Not surprisingly, patient willingness to accept invasive therapy is related directly to tinnitus severity and treatment benefit magnitude. ${ }^{9}$ For patients with mild tinnitus who are unlikely to accept invasive therapy, they are nonetheless likely to accept incision-free therapy. In a cohort of 101 firefighters with mild tinnitus who were surveyed for their willingness to accept implant-free focally destructive Gamma Knife radiosurgery to a single caudate nucleus, $43 \%$ were likely to accept therapy if tinnitus would be improved by $75 \% .{ }^{10}$ The potential treatment population who would accept implant-free lesion therapy, such as radiosurgery or FUS, appears to be substantial. It would seem that patients with tinnitus of even mild severity would rather not be troubled by their auditory phantom, provided the treatment is safe and mostly effective. We are unaware of any implant-free phase I clinical trial targeting the caudate nucleus at this time.

Retaining the capability to explore a variety of stimulation parameter sets conferred by invasive DBS without artificial time constraints was especially important in this early clinical trial to evaluate safety and efficacy. The success of early-phase studies in novel disorders and treatment targets may be negatively impacted by predetermined time limits placed on this discovery process. Triggered adverse events, including visual phantoms, were controllably reversible by changing stimulation parameters. The iterative search method to determine optimal stimulation parameters was unexpectedly lengthy, giving rise to consideration of a closed-loop method akin to betaband activity adaptive stimulation in PD. ${ }^{6,7,13}$ It should be noted that the key distinction between tinnitus and PD is that the former is a sensory phantom perceptual disorder in which multiple sound-quality dimensions impact severity (loudness, rhythm, pitch, location, etc.), and none are accessible to an external observer for objective measurement. The identification of a neurophysiological biomarker for closed-loop adaptive stimulation in tinnitus will be significantly more challenging.

Tinnitus $^{3}$ is a particular class of sensory phantom perceptual disorders, in which elemental, fragmented, or distorted sensations attributable to specific body parts are perceived in the absence of physical triggers. Sensory phantoms typically arise following partial or complete functional amputation of the referred corporeal source. Some well-known examples are phantom limb sensation in extremity amputation, ${ }^{8,11}$ Charles Bonnet syndrome in vision loss, ${ }^{1}$ phantosmia in smell impairment, ${ }^{2}$ and phantom breast syndrome in mastectomized women. ${ }^{12,14}$ This work suggests that the caudate nucleus is deserving of deeper study not only in its promise to treat tinnitus, but also in its potential to mitigate other classes of sensory phantoms.

\section{References}

1. Andreotti AM, Goiato MC, Pellizzer EP, Pesqueira AA, Guiotti AM, Gennari-Filho H, et al: Phantom eye syndrome: a review of the literature. ScientificWorldJournal 2014:686493, 2014

2. Bainbridge KE, Byrd-Clark D, Leopold D: Factors associated with phantom odor perception among US adults: findings from the National Health and Nutrition Examination Survey. JAMA Otolaryngol Head Neck Surg 144:807-814, 2018 
3. De Ridder D, Elgoyhen AB, Romo R, Langguth B: Phantom percepts: tinnitus and pain as persisting aversive memory networks. Proc Natl Acad Sci U S A 108:8075-8080, 2011

4. Hinkley LB, Mizuiri D, Hong O, Nagarajan SS, Cheung SW: Increased striatal functional connectivity with auditory cortex in tinnitus. Front Hum Neurosci 9:568, 2015

5. Larson PS, Cheung SW: Deep brain stimulation in area LC controllably triggers auditory phantom percepts. Neurosurgery 70:398-406, 2012

6. Little S, Beudel M, Zrinzo L, Foltynie T, Limousin P, Hariz $\mathrm{M}$, et al: Bilateral adaptive deep brain stimulation is effective in Parkinson's disease. J Neurol Neurosurg Psychiatry 87:717-721, 2016

7. Little S, Brown P: The functional role of beta oscillations in Parkinson's disease. Parkinsonism Relat Disord 20 (Suppl 1):S44-S48, 2014

8. Nikolajsen L, Jensen TS: Phantom limb pain. Br J Anaesth 87:107-116, 2001

9. Perez PL, Wang SS, Heath S, Henderson-Sabes J, Mizuiri D, Hinkley LB, et al: Human caudate nucleus subdivisions in tinnitus modulation. J Neurosurg [epub ahead of print February 8, 2019. DOI: 10.3171/2018.10.JNS181659]
10. Pross SE, Allen CA, Hong OS, Cheung SW: Willingness-toaccept Gamma Knife radiosurgery for tinnitus among career San Francisco firefighters. Otol Neurotol 35:1026-1032, 2014

11. Ramachandran VS, Hirstein W: The perception of phantom limbs. The D. O. Hebb lecture. Brain 121 (Pt 9):1603-1630, 1998

12. Ramesh, Shukla NK, Bhatnagar S: Phantom breast syndrome. Indian J Palliat Care 15:103-107, 2009

13. Swann NC, de Hemptinne C, Thompson MC, Miocinovic S, Miller AM, Gilron R, et al: Adaptive deep brain stimulation for Parkinson's disease using motor cortex sensing. J Neural Eng 15:046006, 2018

14. Tasmuth T, von Smitten K, Kalso E: Pain and other symptoms during the first year after radical and conservative surgery for breast cancer. Br J Cancer 74:2024-2031, 1996

INCLUDE WHEN CITING

Published online September 24, 2019; DOI: 10.3171/2019.5.JNS191204. 